\title{
Adaptive suppression of power line interference in ultra- low field magnetic resonance imaging in an unshielded environment
}

Xiaolei Huang ${ }^{\mathrm{a}, \mathrm{b}, \mathrm{d}, \mathrm{e}}$, Hui Dong, ${ }^{\mathrm{a}, \mathrm{b}, \mathrm{d}, *}$, Yang Qiue ${ }^{\mathrm{a}, \mathrm{b}, \mathrm{d}, \mathrm{f}}$, Bo $\mathrm{Li}^{\mathrm{a}, \mathrm{b}, \mathrm{d}, \mathrm{f}}$, Quan Tao ${ }^{\mathrm{a}, \mathrm{b}, \mathrm{c}, \mathrm{d}}$, Yi Zhang $^{\mathrm{c}, \mathrm{d}}$, Hans-Joachim Krause ${ }^{\mathrm{c}, \mathrm{d},{ }^{*}}$, Andreas Offenhäusser, ${ }^{\mathrm{c}, \mathrm{d}}$ and Xiaoming Xie ${ }^{\mathrm{a}, \mathrm{b}, \mathrm{d}}$

${ }^{a}$ State Key Laboratory of Functional Materials for Informatics, Shanghai Institute of Microsystem and Information Technology (SIMIT), Chinese Academy of Sciences (CAS), Shanghai 200050, China

b CAS Center for ExcelleNce in Superconducting Electronics (CENSE), Shanghai 200050, China

c Institute of Complex Systems (ICS-8), Forschungszentrum Jülich (FZJ), D-52425 Jülich, Germany

d Joint Research Institute on Functional Materials and Elelctronics, Collaboration between SIMIT and FZJ

e University of Chinese Academy of Sciences, Beijing 100049, China

${ }^{\mathrm{f}}$ China Jiliang University, Hangzhou 310018, China

E-mails: donghui@mail.sim.ac.cn

Address: Shanghai Institute of Microsystem and Information Technology, Chinese Academy of Sciences, Shanghai 200050, China

Tel/Fax: +86 2169976816

h.-j.krause@fz-juelich.de

Address: Institute of Complex Systems (ICS-8), Forschungszentrum Jülich, D-52425

Jülich, Germany

Tel: +49 2461-61-2955

Fax: +49 2461-61-2630 


\begin{abstract}
Power-line harmonic interference and fixed-frequency noise peaks may cause stripeartifacts in ultra-low field (ULF) magnetic resonance imaging (MRI) in an unshielded environment and in a conductively shielded room. In this paper we describe an adaptive suppression method to eliminate these artifacts in MRI images. This technique utilizes spatial correlation of the interference from different positions, and is realized by subtracting the outputs of the reference channel(s) from those of the signal channel(s) using wavelet analysis and the least squares method. The adaptive suppression method is first implemented to remove the image artifacts in simulation. We then experimentally demonstrate the feasibility of this technique by adding three orthogonal superconducting quantum interference device (SQUID) magnetometers as reference channels to compensate the output of one $2^{\text {nd }}$-order gradiometer. The experimental results show great improvement in the imaging quality in both 1D and 2D MRI images at two common imaging frequencies, $1.3 \mathrm{kHz}$ and $4.8 \mathrm{kHz}$. At both frequencies, the effective compensation bandwidth is as high as $2 \mathrm{kHz}$. Furthermore, we examine the longitudinal relaxation times of the same sample before and after compensation, and show that the MRI properties of the sample did not change after applying adaptive suppression. This technique can effectively increase the imaging bandwidth and be applied to ULF MRI in both an unshielded environment and a shielded room made from aluminum sheets.
\end{abstract}

\title{
Keywords:
}

Ultra-low field, magnetic resonance imaging, superconducting quantum interference device, noise compensation, power-line harmonics interference 


\section{Introduction}

As a non-invasive medical imaging method, magnetic resonance imaging (MRI) exhibits excellent soft tissue contrast and is widely used in clinical diagnosis and research. Most commercial MRI systems utilize tesla-range superconducting magnets to obtain high signal-to-noise ratio (SNR) and spatial resolution. Meanwhile, MRI at low/ultra-low field (LF/ULF) attracts people's interest. At LF/ULF, some techniques were developed to improve SNR. For example, a pre-polarization technique involving a current-pulsed coil [1] and an ultra-sensitive detector superconducting quantum interference device (SQUID) were combined to build a low-cost MRI system with acceptable SNR [2,3]. The first SQUID-based ultra-low field (ULF) MRI system was built by the Clarke group at Berkeley, and operated at $132 \mu \mathrm{T}$ [4]. Hyperpolarization techniques, which significantly enhance spin population difference, were introduced in LF/ULF nuclear magnetic resonance (NMR) and MRI systems to obtain stronger SNR [5-7]. Some potential applications of ULF MRI have been demonstrated compared with high field MRI, e.g., enhanced contrast between cancerous and surrounding tissues $[8,9]$, the possibility of imaging in the presence of metallic objects [10], the hybrid biomagnetic imaging of MRI and magnetoencephalography (MEG) [11,12] and the feasibility of neuronal current imaging [13-15]. Most of these advantages were achieved in a magnetically shielded room (MSR), which is costly, or in a shielded room made from aluminum a few millimeters thick. Crucially, the use of any shielded room makes the ULF-MRI system immobile.

An unshielded, portable and inexpensive ULF-MRI system is attractive for, e.g. routine examination in underdeveloped countries. A practical implementation utilizing a Faraday coil as the detector for capsicum imaging in the earth's magnetic field was presented in 2006 [16]. Au unshielded 7-channel SQUID system was implemented by Espy et al. in 2015 [17]. We began studying unshielded systems in an urban laboratory environment in 2008 [18]. In an open environment, temporal magnetic field fluctuations up to $1500 \mathrm{nT}$ affect the stability of the static measurement field $\left(B_{0}\right)$, thus introducing artifacts in the MRI images. We developed an active compensation technique based on low-frequency $(<10 \mathrm{~Hz}$ ) magnetic field spatial correlation and suppressed the fluctuation to $\sim 10 \mathrm{nT}$ [19]. Furthermore, to compensate the spatial inhomogeneity of the environmental magnetic field, which degrades $B_{0}$ as well as the imaging gradient fields, a full-tensor gradient field shimming system was introduced to balance the environment gradient tensor [20]. These two techniques made MRI possible in an urban environment, leading us to construct a 4-channel unshielded system for such applications in 2015 [21]. However, power-line harmonic interference and fixedfrequency noise peaks constitute a problem both in unshielded environment and in a shielded room, faced both by SQUID and inductive detection [16, 22]. For instance, in vivo human brain imaging using SQUIDs [23] and coils [24] as detectors, and lung imaging based on hyperpolarized gases in a conductively shielded room [25] may all endure the power-line harmonic interference. These interference mechanisms introduce stripe-artifacts in MRI images, reducing the image quality and narrowing the signal band. To remove power-line noise from the recorded signal, there are several effective methods: notch filters [26], adaptive filter [27] and reference noise [28]. For example, 
notch filters were used to remove power-line harmonics noise from NMR spectra under earth's magnetic field [29]. However, all the three methods are only appropriate in the single-frequency signal situation. To compensate the interference in a signal frequency range covering several to hundreds of $\mathrm{Hz}$, a method based on adding reference channels was developed for magnetocardiography (MCG) [30]. Note that the signal frequency range of MCG is typically $<50 \mathrm{~Hz}$, far below the typical proton Larmor frequencies $\left(f_{\mathrm{L}}\right)$ of ULF MRI. The reference channels were once introduced into ULF MRI in unshielded environment, but no detailed discussion of the post-processing was given [31].

In this paper, we suggest an adaptive suppression technique to remove artifacts in images caused mainly by strong power-line harmonic interference in an unshielded urban environment. The concept is based on the spatial correlation of the interference terms from different positions. By adding reference channels to our ULF-MRI system, the power-line harmonics are simultaneously recorded by the second-order gradiometer as well as the reference channels. The MRI signals, however, are picked up only by the signal channel. Using wavelet analysis and the least squares method, we are able to subtract the power-line harmonics from the signal channel. Firstly, we verify the method on removing the noise in homogeneous sample image of simulation. We then compare the 1D and 2D MRI images as well as the measured values of spin-lattice relaxation time $\left(T_{1}\right)$ before and after introducing interference suppression at different Larmor frequencies.

\section{Principle of Adaptive Suppression}

\subsection{Spatial correlation and detector configurations}

The basis of the power-line harmonic interference adaptive suppression is the spatial correlation between each signal channel and reference channel in the $\mathrm{kHz}$ frequency range. Generally, people use $2^{\text {nd }}$-order gradiometers in unshielded environment to suppress both homogeneous field noise of distant sources and $1^{\text {st }}$-order gradient noise. In contrast, the different configurations of detectors such as a SQUID magnetometer, a $1^{\text {st }}$-order and a $2^{\text {nd }}$-order axial gradiometers can all be used as reference channels. So we first constructed a three-channel system consisting of a magnetometer in vertical direction, a $1^{\text {st }}$-order gradiometer, and a $2^{\text {nd }}$-order gradiometer. The three channels picked up ambient magnetic field noise and gradient noise in our laboratory. Fig. 1 shows good correlation among the sensors between 3 and $6 \mathrm{kHz}$, where the frequencies of the harmonics are of course identical and the relative amplitudes of the harmonics from each sensor are quite similar. This measurement demonstrates that in our laboratory, the three kinds of configurations for reference channel(s) can be introduced into our unshielded ULF-MRI system. 


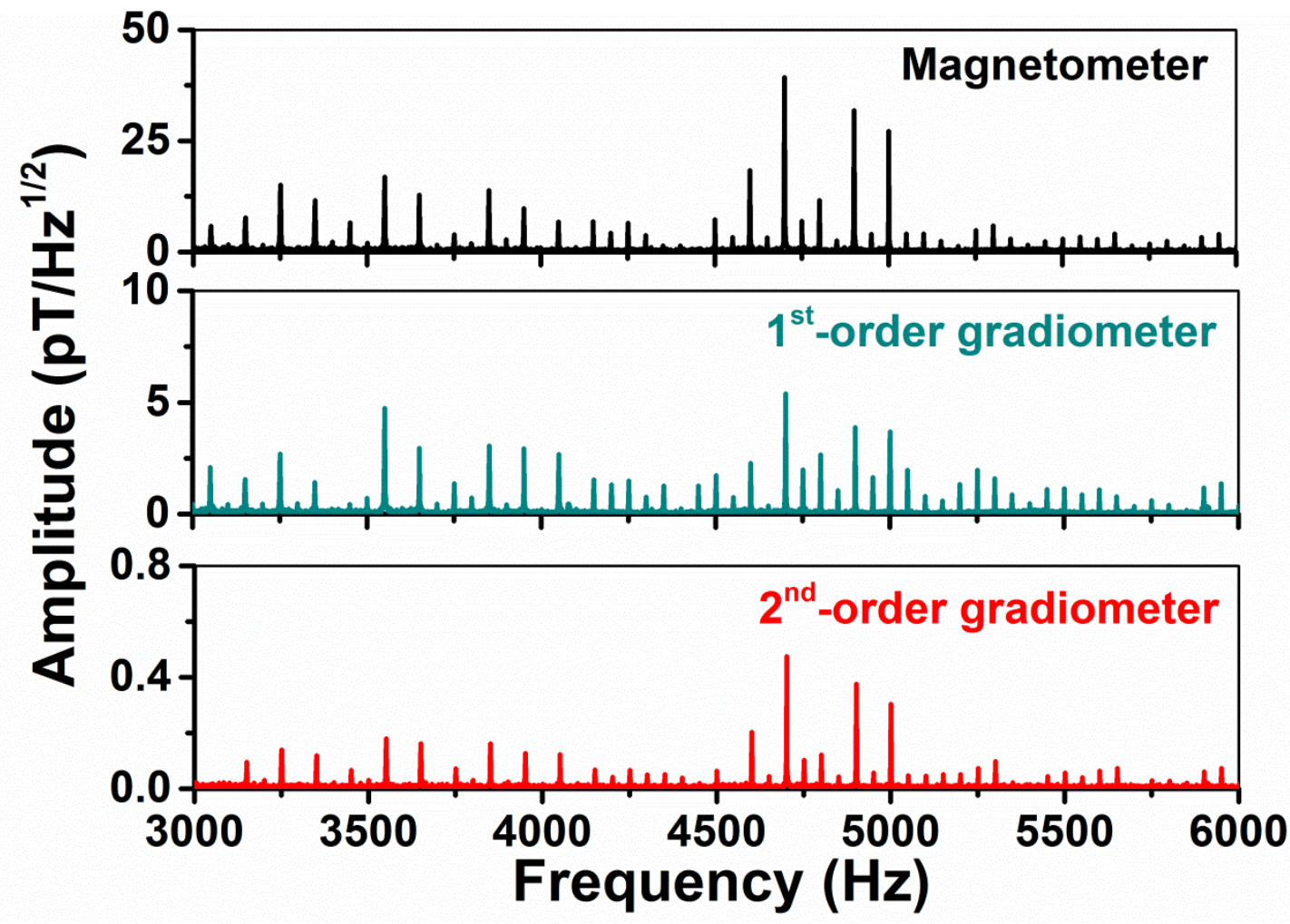

Fig. 1. Ambient noise spectra measured by a magnetometer, a $1^{\text {st }}$-order gradiometer and a $2^{\text {nd }}$-order gradiometer. The loop diameter and the baselines of both gradiometers are $22 \mathrm{~mm}$ and $50 \mathrm{~mm}$, respectively. The loop diameter of the magnetometer is $2 \mathrm{~mm}$. All three sensors are hand wound using $80 \mu \mathrm{m}$ Nb wire.

In unshielded ULF-MRI systems, the images are normally acquired by a $2^{\text {nd }}$-order gradiometer with a vertical axis. The imbalance of a practical $2^{\text {nd }}$-order gradiometer causes a residual response to fluctuations in magnetic field and its $1^{\text {st }}$-order vertical gradient [32], so that the voltage output of a practical $2^{\text {nd }}$-order gradiometers can be described as:

$V_{\text {out }}{ }^{(2)}=\mathrm{k}_{1} B_{\mathrm{x}}{ }^{(2)}+\mathrm{k}_{2} B_{y}{ }^{(2)}+\mathrm{k}_{3} B_{z}{ }^{(2)}+\mathrm{k}_{4} G_{1}{ }^{(2)}+\mathrm{k}_{5} G_{2}{ }^{(2)}$

Here, $B_{\mathrm{x}}{ }^{(2)}, B_{\mathrm{y}}{ }^{(2)}$ and $B_{\mathrm{z}}{ }^{(2)}$ are the three orthogonal components of magnetic field fluctuations, $G_{1}{ }^{(2)}$ and $G_{2}{ }^{(2)}$ the $1^{\text {st }}$ - and $2^{\text {nd }}$-order gradients along the vertical direction, and $\mathrm{k}_{1}$ to $\mathrm{k}_{5}$ the corresponding transfer coefficients. In our laboratory, the static $1^{\text {st }}$-order gradient field in vertical direction is measured to be below $5 \mu \mathrm{T} / \mathrm{m}$ in the system area, and its fluctuations are much less than $0.1 \mu \mathrm{T} / \mathrm{m}$ over a 24 -hour period. The $1^{\text {st }}$-order gradient imbalance of the $2^{\text {nd }}$-order gradiometer is typically $1 \%$, which causes less than a $10-m V$ fluctuation in the SQUID output. Therefore, the term $\mathrm{k}_{4} G_{1}{ }^{(2)}$ can be ignored.

Consequently, in our following experiments, we introduced only three orthogonal magnetometers to compensate the response of $2^{\text {nd }}$-order gradiometer to the three orthogonal components of the magnetic field of the power-line harmonics. Another advantage of using magnetometers instead of gradiometers as reference channels is that the magnetometers have a small size that reduces liquid helium evaporation. 


\subsection{Adaptive suppression algorithm}

For noise suppression at a fixed frequency, the spectral subtraction method in speech signal processing [33] and the aforementioned three methods [26-28] have been developed. However, speech signal processing is performed in the frequency domain, and the methods in [26-28] are only appropriate in the single-frequency situation. We therefore designed an adaptive suppression to suppress fixed-frequency interference in the signal frequency range covering several hundreds of $\mathrm{Hz}$.

There are three key factors we should take into account for the suppression of the power-line harmonics. The first one is the sample Larmor frequency at ULF, typically $1 \sim 10 \mathrm{kHz}$. The second is the image bandwidth $\mathrm{B}_{\mathrm{W}}$, which depends on the size of sample and the imaging gradient field strengths, typically about $800 \sim 900 \mathrm{~Hz}$ for human brain under $100 \mu \mathrm{T} / \mathrm{m}$ gradient field. The third is the noise distribution. There are two harmonics per $100 \mathrm{~Hz}$, if the power-line net frequency is $50 \mathrm{~Hz}$. When $\mathrm{B}_{\mathrm{w}}$ of the sample is more than $50 \mathrm{~Hz}$, the harmonics interference will overlap with the image. In consideration of these factors, we derive an adaptive suppression method to eliminate the noise interference and retain the MRI signal. The signal acquisition is performed in each phase encoding step and then the image is reconstructed using Fourier imaging method after all phase encoding steps finish. For simplicity, we first discuss the signal processing of the adaptive suppression algorithm in one phase encoding step. In the current configuration of the cryogenic probe, the $2^{\text {nd }}$-order gradiometer as the signal channel measures the MRI (spin-echo) signal and the noise simultaneously, while the three reference channels pick up only noise. The sampling rate of all channels is 100 $\mathrm{kSa} / \mathrm{s}$ and the data acquisition time is $100 \mathrm{~ms}$. In this way, the output of the $2^{\text {nd }}$-order gradiometer in each phase encoding step $B_{\text {sig }}(\mathrm{j})$ can be written as:

$B_{\text {sig }}(\mathrm{j})=\mathrm{S}(\mathrm{j})+\mathrm{N}_{2 \mathrm{G}}(\mathrm{j}) \quad \mathrm{j} \in[1,10000]$

(2)

where $\mathrm{j}$ is the number of data points ranging from 1 to $10000, S(j)$ the spin echo signal, $\mathrm{N}_{2 \mathrm{G}}(\mathrm{j})$ the noise picked up by the $2^{\text {nd }}$-order gradiometer. All outputs are in units of voltage.

The key process of the adaptive suppression algorithm is to remove $\mathrm{N}_{2 \mathrm{G}}(\mathrm{j})$ from $B_{\text {sig }}(\mathrm{j})$ and can be accomplished through two steps. Firstly, $B_{\text {sig }}(\mathrm{j})$ is decomposed into different levels in time domain using 1D discrete wavelet analysis (DWA) [34]. Each level corresponds to a frequency range and all levels cover the frequency range of $B_{\text {sig }}(\mathrm{j})$ (50 kHz in our case) which depends on the sampling rate. We assume that the signal levels that cover the frequency range $f_{\mathrm{L}} \pm \frac{1}{2} \mathrm{~B}_{\mathrm{w}}$ are the levels ranging from $\mathrm{n}_{1}$ to $\mathrm{n}_{2}$. The adaptive suppression only takes into account the signal levels and no data processing is performed to the levels with numbers $<\mathrm{n}_{1}$ or $>\mathrm{n}_{2}$. Fig. 2 shows a 
schematic of the signal levels that cover the frequency range $f_{\mathrm{L}} \pm \frac{1}{2} \mathrm{~B}_{\mathrm{w}}$. Because the frequencies and the amplitudes of the harmonics peaks vary greatly in the range of $f_{\mathrm{L}} \pm$ $\frac{1}{2} B_{w}$, the frequency range of each signal level can be different depending on the practical situation. The decomposition of the signal into different levels may help us to process the noise peaks independently and to improve the noise suppression effect. For the $\mathrm{n}_{1}$ level, the noise peaks in the signal channel were suppressed by using the noise from the same levels in the outputs of the reference channels (the detailed process is given in the second step below), without being affected by the noise in the other levels. According to the known Larmor frequency and $\mathrm{B}_{\mathrm{w}}$ of the sample, the $\mathrm{i}^{\text {th }}$ signal level can be written as follows:

$B_{\text {sig }}^{\mathrm{i}}(\mathrm{j})=\mathrm{S}^{\mathrm{i}}(\mathrm{j})+\mathrm{N}_{2 \mathrm{G}}^{\mathrm{i}}(\mathrm{j}) \quad \mathrm{j} \in[1,10000]$

where $\mathrm{n}_{1}<i<\mathrm{n}_{2}$.

Similarly, the outputs of the three orthogonal magnetometers of the $i^{\text {th }}$ level, which only contain noise, can be analyzed as $B_{x, y, z}^{i}(j)$ (a simplified representation of $B_{x}^{i}(j)$, $B_{y}^{i}(j)$ and $\left.B_{z}^{i}(j)\right)$.

The second step of the adaptive suppression is to restrain the harmonics noise with the least squares method. We assume the spin echo contain $\mathrm{m}_{2}$ data points, ranging from $m_{1}+1$ to $m_{1}+m_{2}\left(1<m_{1}, m_{1}+m_{2}<10000\right)$. So there are no MRI signal before $\mathrm{m}_{1}$ or after $\mathrm{m}_{1}+\mathrm{m}_{2}$ data points. The suppression coefficients $\mathrm{K}_{\mathrm{x}, \mathrm{y}, \mathrm{z}}^{\mathrm{i}}$ of the $\mathrm{i}^{\text {th }}$ level can then be obtained by fitting $B_{\text {sig }}^{\mathrm{i}}(\mathrm{j})$ and $\mathrm{B}_{\mathrm{x}, \mathrm{y}, \mathrm{z}}^{\mathrm{i}}(\mathrm{j})$ using the data points ranging from 1 to $m_{1}$ (or from $m_{1}+1$ to 10000 ) based on the least squares method:

$\sum_{j=1}^{m_{1}}\left[B_{\text {sig }}^{i}(j)-K_{x, y, z}^{i} \cdot B_{x, y, z}^{i}(j)\right]^{2} \rightarrow \min$

The derivation of $K_{x, y, z}^{i}$ is not influenced by the spin-echo signal. Then we use $K_{x, y, z}^{i}$ to compensate the noise in the $\mathrm{i}^{\text {th }}$ level:

$B_{\text {comp }}^{i}(\mathrm{j})=B_{\text {sig }}^{\mathrm{i}}(\mathrm{j})-\mathrm{K}_{\mathrm{x}, \mathrm{y}, \mathrm{z}}^{\mathrm{i}} \cdot \mathrm{B}_{\mathrm{x}, \mathrm{y}, \mathrm{z}}^{\mathrm{i}}(\mathrm{j}) \quad \mathrm{j} \in[1,10000]$

The matrix $\mathrm{K}_{\mathrm{x}, \mathrm{y}, \mathrm{z}}^{\mathrm{i}}$ derived from the $\mathrm{i}^{\text {th }}$ level is composed of fixed suppression coefficients $\mathrm{K}_{\mathrm{x}}^{\mathrm{i}}, \mathrm{K}_{\mathrm{y}}^{\mathrm{i}}$ and $\mathrm{K}_{\mathrm{z}}^{\mathrm{i}}$. So the compensated outputs $B_{\text {comp }}{ }^{\mathrm{n}_{1}}(\mathrm{j}), \ldots$, $B_{\text {comp }}{ }^{\mathrm{n}_{2}}(\mathrm{j})$ of the signal channel from the $\mathrm{n}_{1}{ }^{\text {th }}$ to $\mathrm{n}_{2}{ }^{\text {th }}$ levels can be described:

$$
\begin{gathered}
\left.\left[\begin{array}{c}
B_{\text {comp }}{ }^{\mathrm{n}_{1}}(\mathrm{j}) \\
\vdots \\
B_{\text {comp }}{ }^{\mathrm{n}_{2}}(\mathrm{j})
\end{array}\right]=\left[\begin{array}{c}
B_{\text {sig }}{ }^{\mathrm{n}_{1}}(\mathrm{j}) \\
\vdots \\
B_{\text {sig }}{ }^{\mathrm{n}_{2}}(\mathrm{j})
\end{array}\right]-\left[\begin{array}{c}
\mathrm{K}_{\mathrm{x}}^{\mathrm{n}_{1}} \\
\vdots \\
\mathrm{K}_{\mathrm{x}}^{\mathrm{n}_{2}}
\end{array}\right] \cdot\left[\begin{array}{c}
B_{\mathrm{x}}^{\mathrm{n}_{1}}(\mathrm{j}) \\
\vdots \\
B_{\mathrm{x}}^{\mathrm{n}_{2}}(\mathrm{j})
\end{array}\right]\right]-\left[\left[\begin{array}{c}
\mathrm{K}_{\mathrm{y}}^{\mathrm{n}_{1}} \\
\vdots \\
\mathrm{K}_{\mathrm{y}}^{\mathrm{n}_{2}}
\end{array}\right] \cdot\left[\begin{array}{c}
B_{\mathrm{y}}^{\mathrm{n}_{1}}(\mathrm{j}) \\
\vdots \\
B_{\mathrm{y}}^{\mathrm{n}_{2}}(\mathrm{j})
\end{array}\right]\right]-\left[\left[\begin{array}{c}
\mathrm{K}_{\mathrm{z}}^{\mathrm{n}_{1}} \\
\vdots \\
\mathrm{K}_{\mathrm{z}}^{\mathrm{n}_{2}}
\end{array}\right] \cdot\left[\begin{array}{c}
B_{\mathrm{z}}^{\mathrm{n}_{1}}(\mathrm{j}) \\
\vdots \\
B_{\mathrm{z}}^{\mathrm{n}_{2}}(\mathrm{j})
\end{array}\right]\right] \\
\mathrm{j} \in[1,10000]
\end{gathered}
$$


The total compensated output of the signal channel of one phase encoding step should be written:

$$
B_{\text {comp }}(\mathrm{j})=\sum_{\mathrm{i}=\mathrm{n}_{1}}^{\mathrm{n}_{2}} B_{\text {comp }} \mathrm{i}(\mathrm{j}) \quad \mathrm{j} \in[1,10000]
$$

For each phase encoding step, the same adaptive suppression process is implemented. The noise-suppressed images are obtained by performing Fourier transform to the compensated outputs of all phase encoding steps.

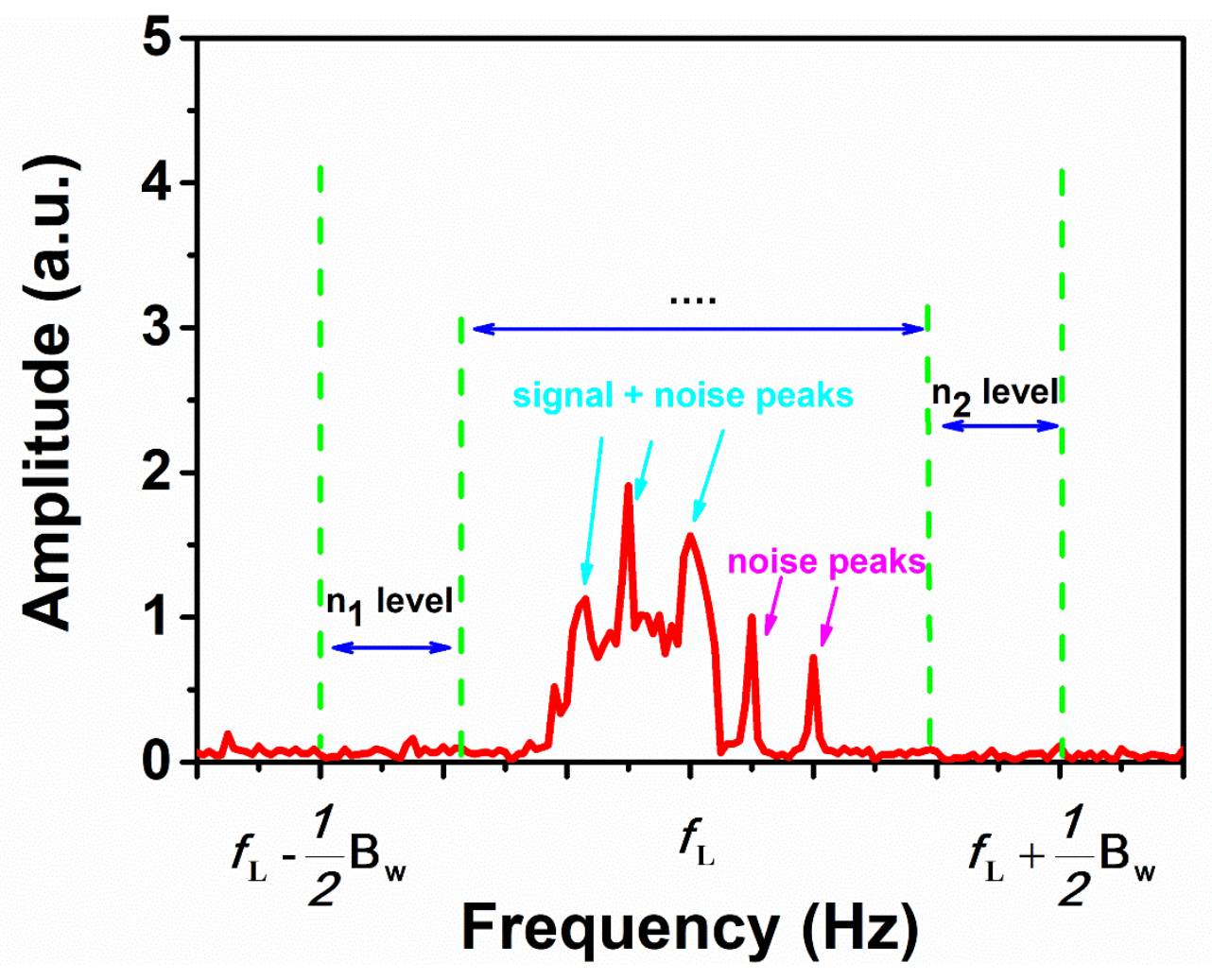

Fig. 2. Schematic of the signal levels that cover the frequency range $f_{\mathrm{L}} \pm \frac{1}{2} \mathrm{~B}_{\mathrm{w}}$. The frequency range of each signal level (from $\mathrm{n}_{1}$ to $\mathrm{n}_{2}$ ) can be different depending on the practical situation. No data processing is performed beyond the range $f_{\mathrm{L}} \pm \frac{1}{2} \mathrm{~B}_{\mathrm{w}}$.

\subsection{Numerical simulation}

We firstly verified the effectiveness of the adaptive suppression using a 2D MRI numerical simulation. A homogeneous rectangular sample with the side lengths of 45 $\mathrm{mm} \times 35 \mathrm{~mm}$ and a $20 \mathrm{~mm} \times 20 \mathrm{~mm}$ square hole at center was used in the simulation, as shown in Fig. 3(a). The signal frequency $f_{\mathrm{L}}$ is $4800 \mathrm{~Hz}$. The frequency encoding gradient was $186 \mu \mathrm{T} / \mathrm{m}$ and the phase encoding gradient was from $-45 \mu \mathrm{T} / \mathrm{m}$ to $+90 \mu \mathrm{T} / \mathrm{m}$ with 31 steps. The 2D imaging resolution was about $1.25 \mathrm{~mm} \times 4 \mathrm{~mm}$ and the imaging field of view (FOV) was $101 \mathrm{~mm} \times 163 \mathrm{~mm}$. To simulate the practical noise performance, we added the noise picked up by the Z-direction magnetometer to the reference image in 31 phase encoding steps. The amplitude 
difference between the added noise and the noise picked up by the magnetometer is determined by the ratio of the effective areas of $2^{\text {nd }}$-order gradiometer to that of the magnetometer [32]. The noise-added MRI image is depicted in Fig. 3(b). The noise of the synthesized signal was then compensated based-on the algorithm described in 2.2. The determination of the optimal mother wavelet depends on the sample structure [35]. We use the Daubechies mother wavelet because of the simple structure of the sample. We can see that the stripe-artifacts are almost eliminated from Fig. 3(c). The residual image defined as the difference between Fig. 3(b) and (c) was introduced to assess the de-noising effectiveness [36], as depicted in Fig. 3(d). Fig. 3(d) shows only noise artifacts and the sample is not observable. It demonstrates that the adaptive suppression method is applicable for retrieving the sample signal from the power-line harmonics.
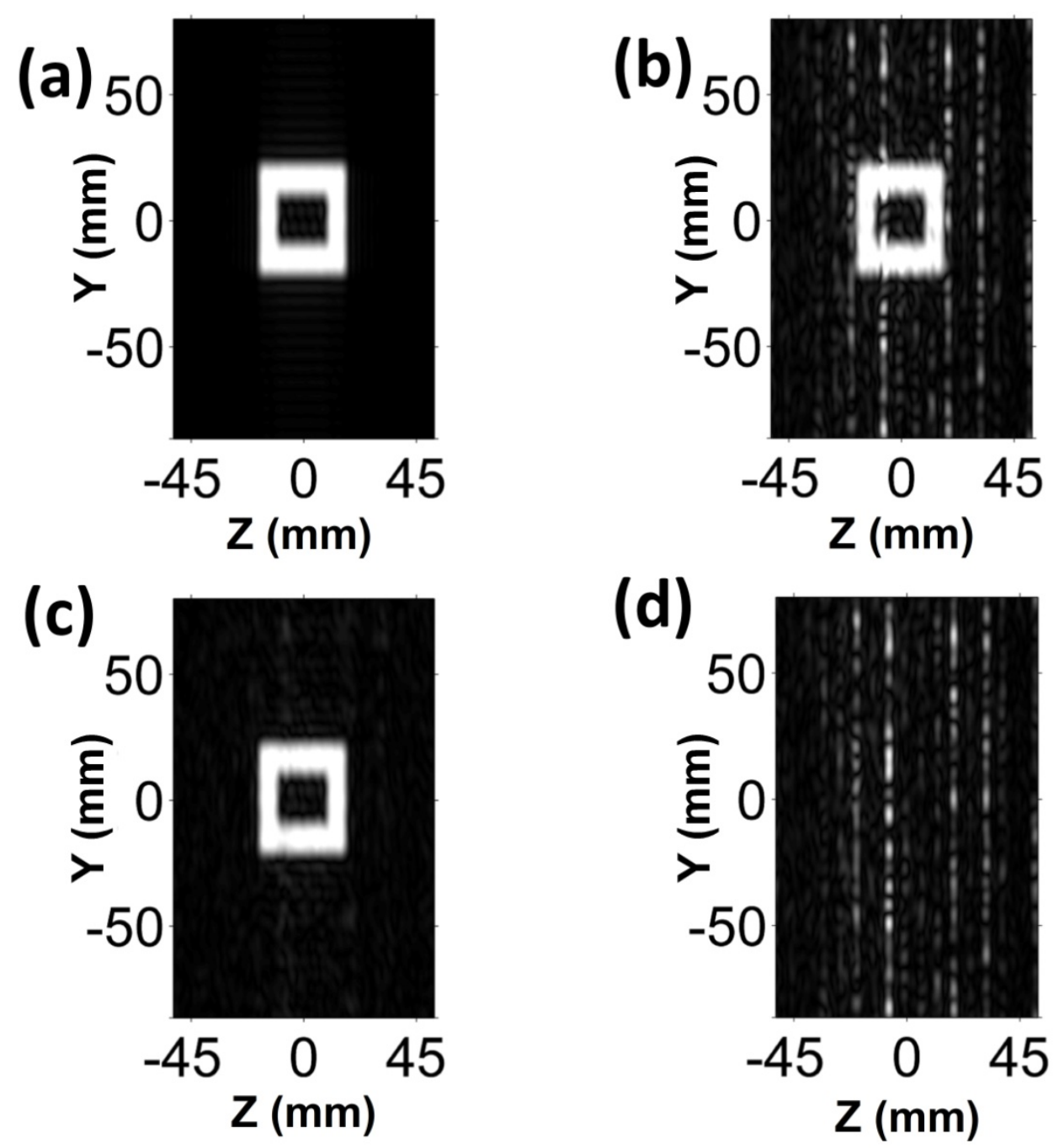

Fig. 3. The simulated 2D MRI images of a rectangular homogeneous sample without noise (a) and with the measured noise (b). (c) The image after adaptive suppression. (d) The residual image defined as the difference between (b) and (c).

\section{System design}

In this section, we describe our cryogenic probe, consisting of one $2^{\text {nd }}$-order 
gradiometer as the signal channel and three orthogonal magnetometers as the reference channels. We also describe the unshielded ULF-MRI system and the imaging protocol.

In the SQUID system, as shown in Fig. 4 , the $2^{\text {nd }}$-order gradiometer is placed at the bottom of the liquid helium dewar for MRI signal detection. The diameter and the baseline of the gradiometers is $22 \mathrm{~mm}$ and $50 \mathrm{~mm}$ and the input-coil inductance of the SQUID is $680 \mathrm{nH}$. To ensure the reference channel picks up only environmental interference, the orthogonal magnetometers, each equipped with a 2-mm diameter, oneturn pick-up coil, are placed about $45 \mathrm{~mm}$ above the lowest coil of the $2^{\text {nd }}$-order gradiometer. The distance between the lowest coil of the $2^{\text {nd }}$-order gradiometer and the room-temperature sample is $15 \mathrm{~mm}$. The top of the four SQUIDs is $210 \mathrm{~mm}$ above the lowest coil of the $2^{\text {nd }}$-order gradiometer. The measured environmental white noise spectrum of the unshielded system above $3 \mathrm{kHz}$ is about $12 \mathrm{fT} / \sqrt{\mathrm{Hz}}$.

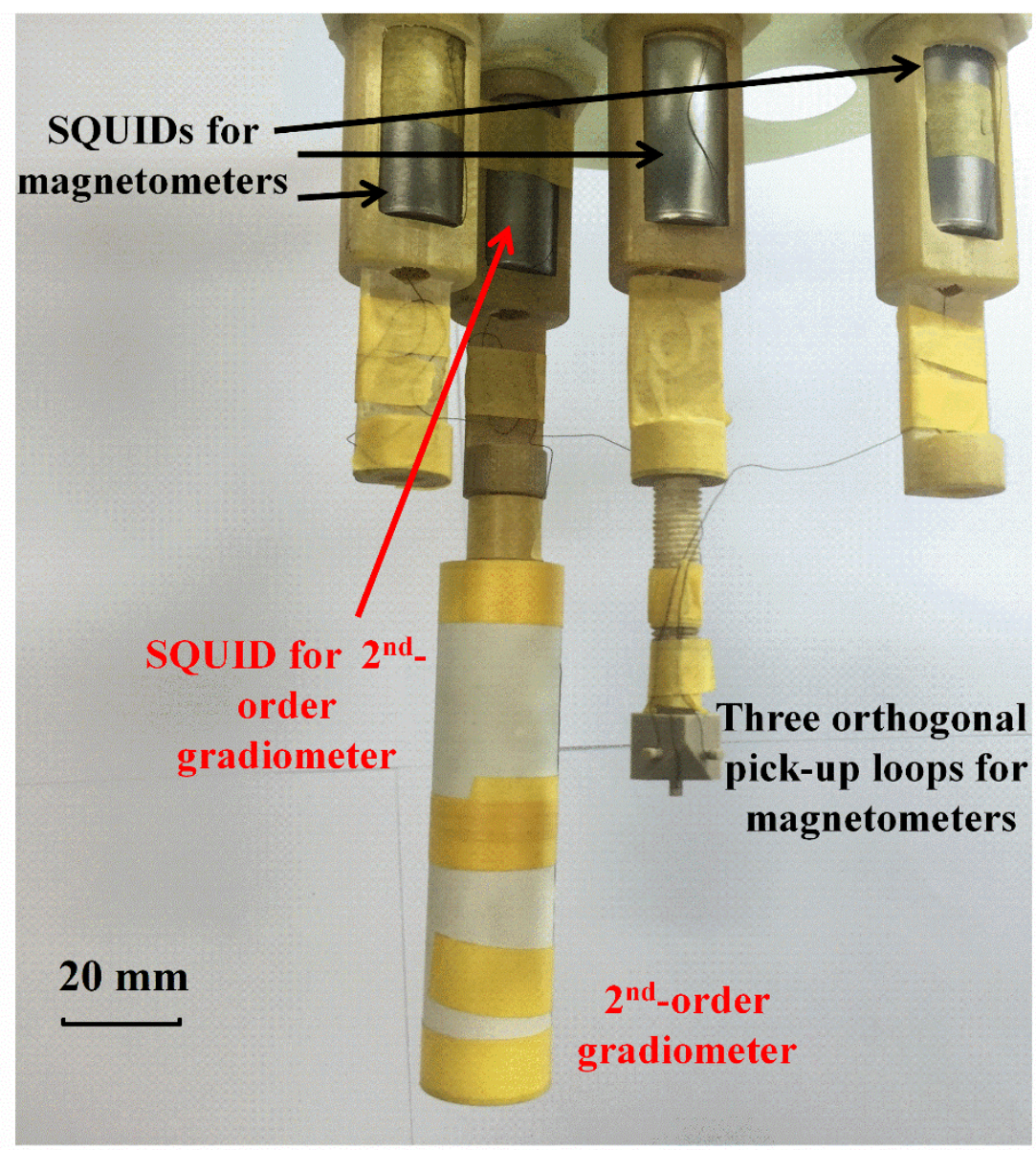

Fig. 4. Photo of the cryogenic probe including four SQUIDs, three orthogonal magnetometers and a $2^{\text {nd }}$-order gradiometer

Our unshielded ULF-MRI system, shown in Fig. 5 (a), consists of the SQUID system, a commercial liquid helium dewar, a transportation system to move the sample from the magnet to the bottom of the dewar and a $0.65 \mathrm{~T}$ permanent magnet pair for sample prepolarization. A detailed description of the system can be found in $[20,21]$.

The 2D Fourier imaging sequence and $T_{1}$ relaxation time measurement sequence are depicted in Fig. 5 (b). After prepolarizing the sample in the gap of the magnets for 
a time $T_{\mathrm{P}}=5 \mathrm{~s}$, the sample was transported to the outer bottom of the dewar in a transportation time $T_{\text {tran }}=550 \mathrm{~ms}$. Then, the $\pi / 2$ and $\pi$ excitation field pulses were applied to activate the nuclear magnetic resonance signal at the Larmor frequency $f_{\mathrm{L}}$. Subsequently, spin echoes were recorded and imaging was performed with the 2D Fourier imaging protocol. The frequency encoding gradient $G_{z}$ was kept on during the measurement, and the phase was encoded with the gradient $G_{\mathrm{y}}$ using $N_{\mathrm{y}}$ steps. The phase encoding time $T_{\mathrm{pe}}$ and the signal acquisition time $T_{\mathrm{a}}$ were $32 \mathrm{~ms}$ and $100 \mathrm{~ms}$, respectively. For the $T_{1}$ relaxation time measurement, a series of $T_{1 \text { deday }}$ times were added before the $\pi / 2$ pulse to fit $T_{1}$ value according to $A=\left(A_{0}-A_{\mathrm{B} 0}\right) e^{-t / T_{1}}+A_{\mathrm{B} 0}$. Here, $A_{0}$ is the initial value of the sample magnetization as the sample arrived at the dewar outer bottom in $B_{0}$ field. $A_{\mathrm{B} 0}$ is the magnetization in $B_{0}$ field in thermal equilibrium. In the $T_{1}$ sequence, the $G_{\mathrm{z}}$ and $G_{\mathrm{y}}$ were set to zero.
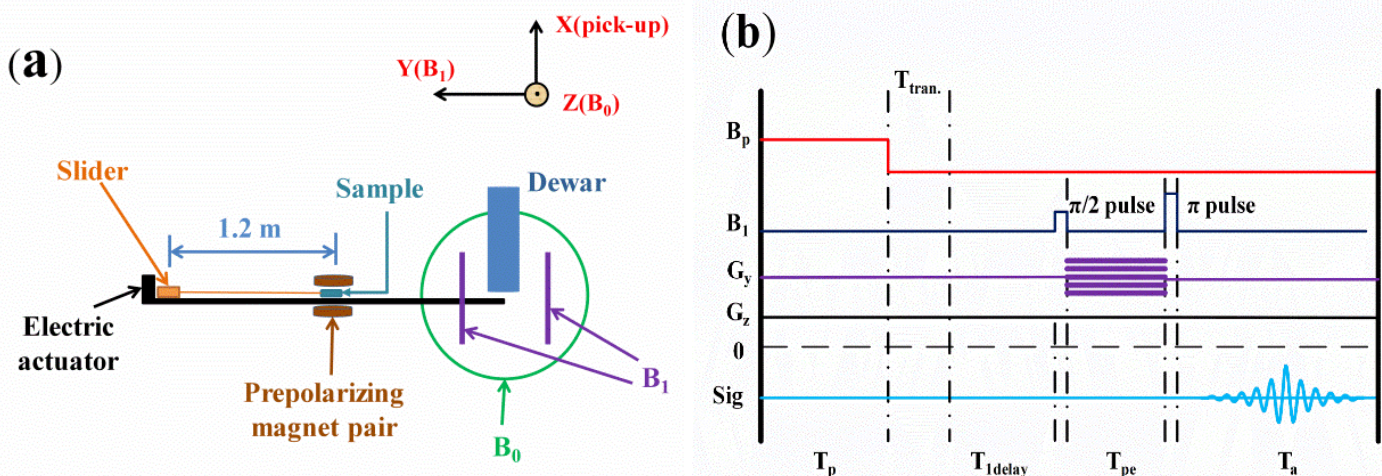

Fig. 5. (a) Schematic of the unshielded ULF-MRI system (gradient coils are not shown in for simplicity). (b) Sequence for $2 \mathrm{D}$ Fourier imaging and $T_{1}$ relaxation time measurement.

\section{Experimental results}

Given the good spatial correlation and the fact that the homogeneous magnetic field components dominate the gradient components in the ambient environment, we implemented $50 \mathrm{~Hz}$ harmonics compensation and reliability demonstration experiments using our ULF-MRI system. In addition, we acquired 1D and 2D MRI images with and without adaptive suppression technique.

\subsection{Effect of Adaptive Suppression on 1D MRI}

In order to verify this method in a wider frequency range, we chose the $1 \mathrm{D}$ imaging frequencies at $1.3 \mathrm{kHz}$ and $4.8 \mathrm{kHz}$, because the $1 \sim 2 \mathrm{kHz}$ range is a typical frequency range of the hybrid MRI-MEG application [11,12], and $4.8 \mathrm{kHz}$ is a common imaging frequency of ULF MRI. To evaluate the robustness of the adaptive suppression in the case of low SNR, we applied frequency encoding gradients in this experiment to make the signal amplitude comparable to those of the noise peaks. The gradient strengths at $1.3 \mathrm{kHz}$ and $4.8 \mathrm{kHz}$ are $3.6 \mu \mathrm{T} / \mathrm{m}$ and $10.35 \mu \mathrm{T} / \mathrm{m}$, respectively. The effect of the suppression is illustrated by the 1D MRI of the water sample in Fig. 6. The noise peaks 
were removed or greatly suppressed, while the amplitudes of the sample signal peaks were preserved. At both $1.3 \mathrm{kHz}$ and $4.8 \mathrm{kHz}$, the 1D MRI signal SNR was substantially increased. Because the amplitude and width of the interference peaks are corresponding to the gray scale and width of stripe-artifacts in the MRI image, it is necessary to calculate the sums of area integral under the harmonic interference peaks before and after compensation. The integral radius is $10 \mathrm{~Hz}$, the same as our frequency resolution. The results are shown in Table 1 . It shown that more than $80 \%$ power of the interference peaks was suppressed within $\pm 500 \mathrm{~Hz}$ centered at the Larmor frequency. $\mathrm{B}_{\mathrm{w}}=1 \mathrm{kHz}$ meets the needs of human brain under $100 \mu \mathrm{T} / \mathrm{m}$ gradient field. In adaptive suppression, we assume that all the $50 \mathrm{~Hz}$ harmonics are from the same source (powerline grid), which can be regarded as far-field interference. Therefore, the $50 \mathrm{~Hz}$ harmonics picked up by the $2^{\text {nd }}$-order gradiometer and the reference channels are correlated and have fixed phases and amplitudes. However, some near-field interference sources may also radiate $50 \mathrm{~Hz}$ harmonics (for example, the equipment near our system). The phases and amplitudes of the near-field interference may change during the signal acquisition time $T_{\mathrm{a}}$. The near-field interference cannot be suppressed by fixed suppression coefficients, which were used in the far-field interference case. The uncertainty of the phases and amplitudes limits the further improvement of the suppression.
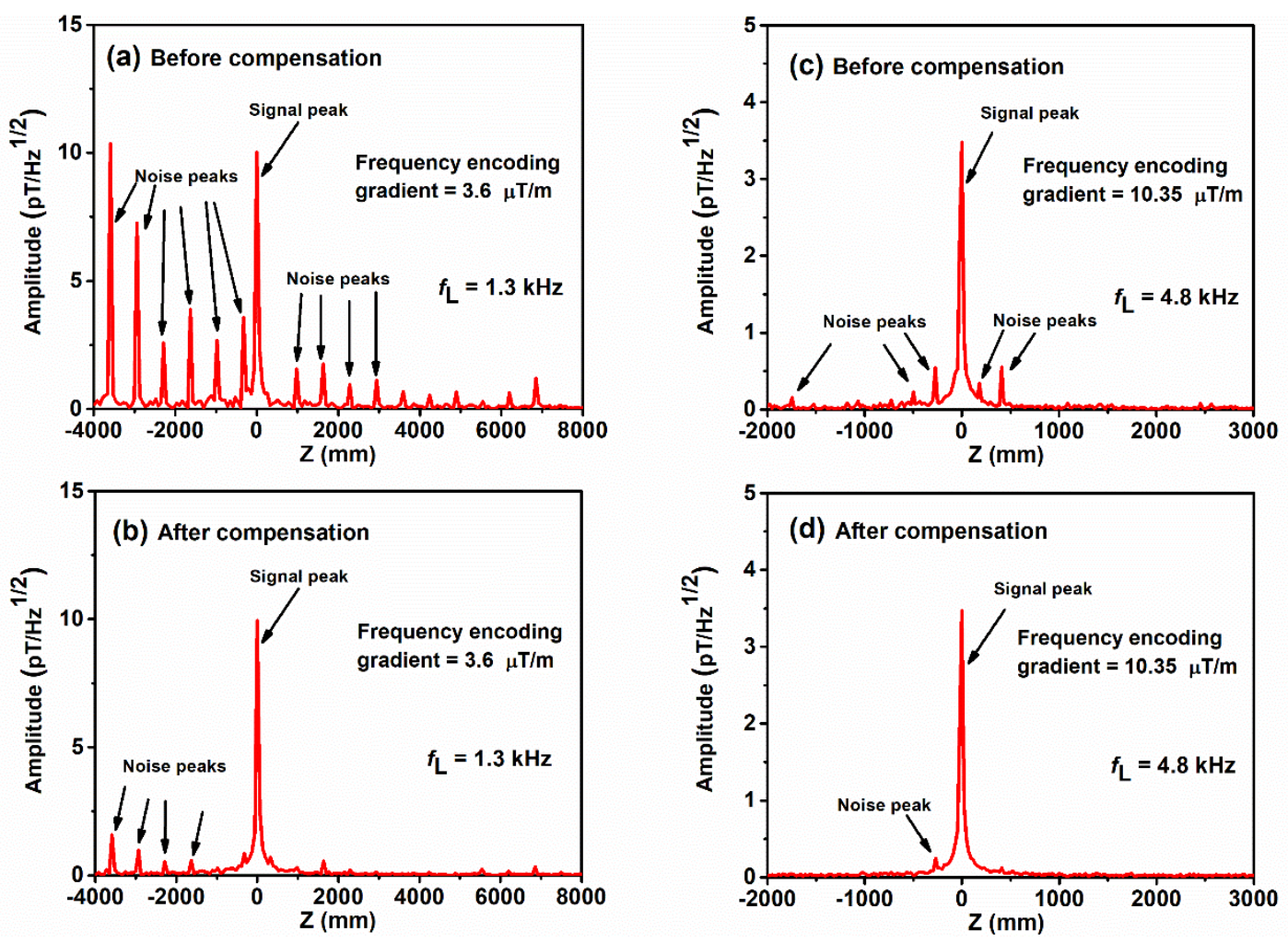

Fig. 6. The 1D water MRI signals (a) before and (b) after applying the adaptive suppression at $1.3 \mathrm{kHz}$. (c) and (d) are the 1D water MRI signals before and after applying the adaptive suppression at $4.8 \mathrm{kHz}$. The position axes were calculated based on the gradient field 
strengths.

\section{Table 1(a)}

Calculated sums of area integral under the harmonics noise peaks before and after the compensation at $1.3 \mathrm{kHz}$

\begin{tabular}{|c|c|c|c|c|}
\hline \multicolumn{2}{|c|}{$\begin{array}{l}\text { Frequency bandwidth of area } \\
\text { integral sum }(\mathrm{Hz}) \\
\text { (center frequency }=1.3 \mathrm{kHz} \text { ) }\end{array}$} & \pm 100 & \pm 200 & \pm 500 \\
\hline \multirow{2}{*}{$\begin{array}{l}\text { Sums of area } \\
\text { integral (pT) }\end{array}$} & $\begin{array}{l}\text { Before } \\
\text { compensation }\end{array}$ & 16.9 & 38.1 & 125.7 \\
\hline & $\begin{array}{l}\text { After } \\
\text { compensation }\end{array}$ & 3.3 & 4.9 & 19.5 \\
\hline \multicolumn{2}{|c|}{$\begin{array}{l}\text { Percentage of interference peaks } \\
\text { suppression after compensation (\%) }\end{array}$} & 80.5 & 87.1 & 84.5 \\
\hline
\end{tabular}

\section{Table 1(b)}

Calculated sums of area integral under the harmonics noise peaks before and after the compensation at $4.8 \mathrm{kHz}$

\begin{tabular}{|c|c|c|c|c|}
\hline \multicolumn{2}{|c|}{$\begin{array}{l}\text { Frequency bandwidth of area } \\
\text { integral sum }(\mathrm{Hz}) \\
\text { (center frequency }=4.8 \mathrm{kHz} \text { ) }\end{array}$} & \pm 100 & \pm 200 & \pm 500 \\
\hline \multirow{2}{*}{$\begin{array}{l}\text { Sums of area } \\
\text { integral (pT) }\end{array}$} & $\begin{array}{l}\text { Before } \\
\text { compensation }\end{array}$ & 8.9 & 17.6 & 23.0 \\
\hline & $\begin{array}{l}\text { After } \\
\text { compensation }\end{array}$ & 1.7 & 3.1 & 3.2 \\
\hline \multicolumn{2}{|c|}{$\begin{array}{l}\text { Percentage of interference peaks } \\
\text { suppression after compensation (\%) }\end{array}$} & 80.9 & 82.4 & 86.1 \\
\hline
\end{tabular}

\subsection{Reliability demonstrated by detecting $T_{1}$ relaxation times}

The longitudinal relaxation time $T_{1}$, transverse relaxation time $T_{2}$ and proton density are essential parameters in MRI which reflect the intrinsic properties of the sample being imaged. An essential requirement of adaptive suppression is that these imaging factors must not change after compensation. In this subsection, we demonstrate the reliability of the adaptive suppression technique by measuring $T_{1}$ of a solution of $\mathrm{CuSO}_{4}$. The measured $T_{1}$ delay step size is $50 \mathrm{~ms}$ with 19 steps. Fig. 7(a) and (b) show the $T_{1}$ curves before and after compensation. The values of $T_{1}$ do not change within the error bars of the measurement, demonstrating that the adaptive suppression conserves the sample signal's intrinsic property in terms of the $T_{1}$ relaxation time. 

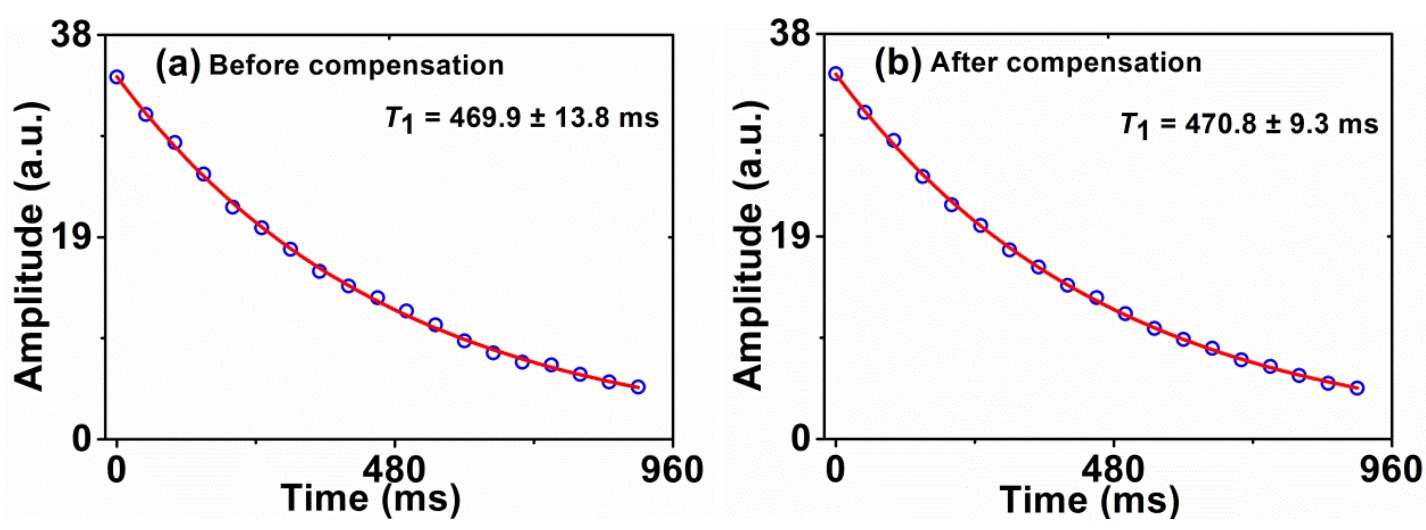

Fig. 7. $T_{1}$ measurement results of $\mathrm{CuSO}_{4}$ solution (a) before and (b) after compensation.

\subsection{Effect of Adaptive Suppression on 2D MRI}

Given the successful demonstration of the numerical simulation and the compensation in 1D MRI spectra, the same method was implemented for 2D MRI images. We set all of the imaging parameters and the size of sample the same as in the simulation. The sample is tap water in a $45 \mathrm{~mm} \times 35 \mathrm{~mm}$ rectangular box with a 20 $\mathrm{mm} \times 20 \mathrm{~mm}$ square hole at center. The uncompensated images in Fig. 8(a) clearly exhibit stripe-artifacts perpendicular to the $\mathrm{Z}$ direction. We determined the coefficient matrix K in Eq.(4) from the output of the $2^{\text {nd }}$-order gradiometer and the magnetometers in each phase encoding step. Fig. 8(b) is the image compensated using only the output of the Z-direction magnetometer; all stripe-artifacts have obviously been suppressed. When we compensated the noise using three magnetometers, the imaging quality shown in Fig. 8(c) was further improved. Comparing Fig. 8(b) and (c), we see that the $50 \mathrm{~Hz}$ harmonics interference along the Z-direction is strongest in our unshielded laboratory environment. The distance between two odd harmonics is $\sim 26 \mathrm{~mm}$. The residual image in Fig. 8(d) reflecting the difference between Fig. 8(a) and (c) does not show the sample shape. To evaluate the suppression effect, we chose two artifacts (line A \& B in Fig. 8(a)) which did not cross the sample image and compare their sums of gray scale values before and after compensation. In Fig. 8(c), lines A and B are suppressed by $86 \%$ and $92 \%$, respectively. Although suppressing the interference using all outputs of the orthogonal magnetometers may significantly improve the image quality, the Z-direction suppression exhibits acceptable improvement. Single-channel compensation decreases the number of SQUIDs and readout electronics and also reduces the complexity of the system. In the future, we may use single-channel or three-channel suppression depending on the actual demand of the MRI experiment. 

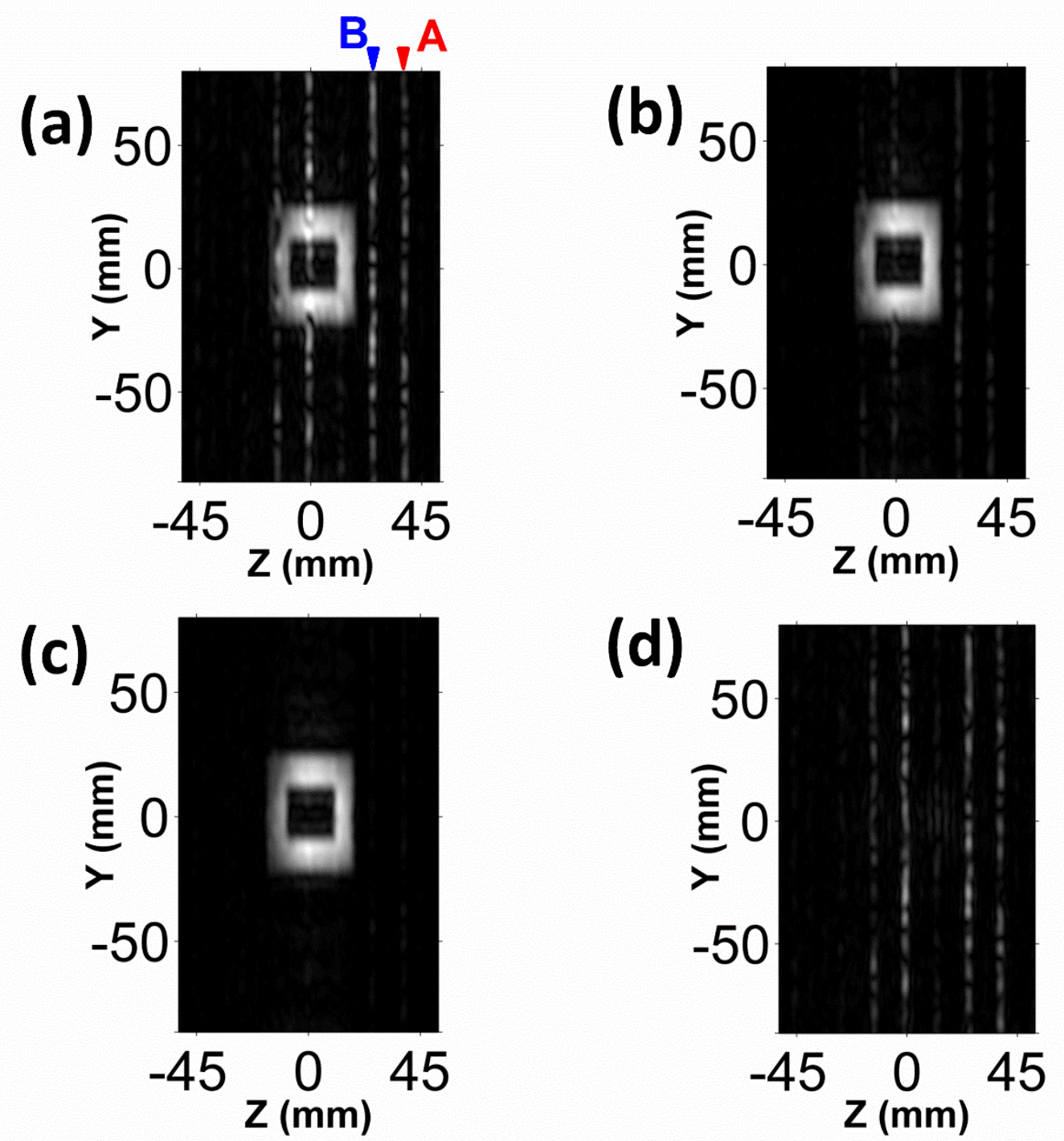

Fig.8. (a) The sample 2D MRI image without compensation. (b) the image after adaptive suppression only in the Z-direction. (c) the image after adaptive suppression in all three directions. (d) the residual image defined as the difference between (a) and (c). Specifically, the artifact lines A and B are suppressed by $86 \%$ and $92 \%$ in (c), respectively.

\section{Conclusions and Discussion}

In this paper, an adaptive suppression method was described combining the reference channels and the adaptive suppression algorithm to eliminate stripe-artifacts in the MRI images caused mainly by environmental power-line harmonic interference. The use of three orthogonal SQUID magnetometers as reference channels may greatly improve the suppression of power-line harmonic peaks picked up by the signal channel in an unshielded urban environment. The results demonstrate that the suppression technique successfully improves the quality of MRI images without changing the measured values of the sample properties. The adaptive suppression can also be applied to ULF MRI in a conductively shielded room when the power-line harmonics 
interference cannot be ignored. The adaptive method is based on spatial correlation of the far-field interference from different positions, so the effect of the adaptive suppression is independent of the choice of imaging detectors. Therefore, traditional Faraday coils can also be used as the reference channels in adaptive suppression for diverse NMR/MRI applications.

\section{Acknowledgements}

The authors gratefully thank Prof. John Clarke and Dr. Shulin Zhang for useful discussions and thoughtful comments on the manuscript. This work was supported in part by the Strategic Priority Research Program (B) of the Chinese Academy of Sciences under Grant XDB04020200 and in part by the National Natural Science Foundation of China under Grant 11204339.

\section{References}

[1] M.E. Packard, R. Varian, Free nuclear induction in the Earth's magnetic field, Phys. Rev. 93 (1954) 941

[2] J. Vrba, J. Nenonen, L. Trahms, Biomagnetism The SQUID Handbook volume II: Applications of SQUIDs and SQUID Systems ed J Clarke and A I Braginski (Weinheim: Wiley-VCH), 2006, pp. 269-390

[3] J. Clarke, M. Hatridge, M. Mosle, SQUID-detected magnetic resonance imaging in microtesla fields, Ann. Rev. Biomed. Eng. 9 (2007) 389 - 413.

[4] R. McDermott, S. Lee, Ten Haken B, A.H. Trabesinger, A. Pines and J. Clarke, Microtesla MRI with a superconducting quantum interference device, Proc. Nat. Acad. Sci. USA 101 (2004):7857-61.

[5] S. Glöggler, R. Müller, J. Colell, M. Emondts, M. Dabrowski, B. Blümich, Para-hydrogen induced polarization of amino acids, peptides and deuterium-hydrogen gas, Phys. Chem. Chem. Phys. 13 (2011):13759-13764.

[6] A. Coffey, K. Kovtunov, D. Barskiy, I. Koptyug, R. Shchepin, K. Waddell, P. He, K. Groome, Q. Best, F. Shi, B. Goodson, E. Chekmenev, High-resolution low-field molecular magnetic resonance imaging of hyperpolarized liquids, Anal. Chem. 86 (2014) 9042-9049.

[7] D.E.J. Waddington, M. Sarracanie, H. Zhang, N. Salameh, D.R. Glenn, E. Rej, T. Gaebel, T. Boele, R.L. Walsworth, D.J. Reilly, M.S. Rosen, Nanodiamond-enhanced MRI via in situ hyperpolarization, Nat. Commun. 8 (2017) 15118.

[8] S. Busch, M. Hatridge, M. Mößle, W. Myers, T. Wong, M. Mück, K. Chew, K. Kuchinsky, J. Simko, J. Clarke, Measurements of $\mathrm{T}_{1}$-relaxation in ex vivo prostate tissue at $132 \mu \mathrm{T}$, Magn. Reson. Med. 67 (2012) 1138-1145.

[9] S. J. Lee, J. H. Shim, K. Kim, S. M. Hwang, K. K. Yu, S. Lim, J.H. Han, H. Yim, J.-H.. Kim, Y.S. Jung, K.S. Kim, $\mathrm{T}_{1}$ relaxation measurement of ex-vivo breast cancer tissues at ultralow magnetic fields, Biomed. Res. Int. 2015 (2015) 385428.

[10] M. Mosle, S.-I. Han, W. Myers, S.K. Lee, N. Kelso, M. Hatridge, A. Pine, J. Clarke, SQUID- 
detected microtesla MRI in the presence of metal, J. Magn. Reson. 179 (2006) 146 - 151.

[11] V.S. Zotev, A.N. Matlashov, P.L. Volegov, I.M. Savukov, M.A. Espy, J.C. Mosher, J.J. Gomez, R.H. Kraus Jr., Microtesla MRI of the human brain combined with MEG, J. Magn. Reson. 194 (2008) $115-120$.

[12] P.T. Vesanen, J.O. Nieminen, K.C. Zevenhoven, J. Dabek, L.T. Parkkonen, A.V. Zhdanov, J. Luomahaara, J. Hassel, J. Penttila, J. Simola, A.I. Ahonen, J.P. Makela, R.J. Ilmoniemi, Hybrid ultra-low-field MRI and magnetoencephalography system based on a commercial whole-head neuromagnetometer, Magn. Reson. Med. 69 (2013) 1795.

[13] R.H. Kraus, P. Volegov, A. Matlachov, M. Espy, Toward direct neural current imaging by resonant mechanisms at ultra-low field, Neuroimage 39 (2008) 310-317.

[14] A.M. Cassará, B. Maraviglia, S. Hartwig, L. Trahms, M. Burghoff, Neuronal current detection with low-field magnetic resonance: simulations and methods, Magn. Reson. Imaging. 27 (2009):1131-1139.

[15] S. Kim, M.C. Stephenson, P.G. Morris, S.R. Jackson, tDCS-induced alterations in GABA concentration within primary motor cortex predict motor learning and motor memory: A 7 T magnetic resonance spectroscopy study, Neuroimage 99 (2014) 237-243.

[16] M.E. Halse, A. Coy, R. Dykstra, C. Eccles, M. Hunter, R. Ward, P.T. Callaghan, A practical and flexible implementation of 3D MRI in the Earth's magnetic field, J. Magn. Reson. 182 (2006) 75-83.

[17] M.A. Espy, P.E. Magnelind, A.N. Matlashov, S.G. Newman, H.J. Sandin, L.J. Schultz, R. Sedillo, A.V. Urbaitis, P.L. Volegov, Progress toward a deployable SQUID-based ultra-low field MRI system for anatomical imaging, IEEE Trans. Appl. Supercond. 25 (2015) 1601705.

[18] H. Dong, Y. Wang, L. Zhang, Y. Sun, X. Xie, Detection of proton NMR signal in the Earth's magnetic field at an urban laboratory environment without shielding, Supercond. Sci. Technol. 20 (2008) 115009

[19] L. Qiu, C. Liu, H. Dong, L. Xu, Y. Zhang, H.-J. Krause, X. Xie, Magnetic Field Improved ULFNMR measurement in an unshielded laboratory using a Low-Tc SQUID, Phys. Procedia. 36 (2012):388-393.

[20] H. Dong, L. Qiu, W. Shi, B. Chang, Y. Qiu, L. Xu, C. Liu, Y. Zhang, H.-J. Krause, A. Offenhäusser, X. Xie, Ultra-low field magnetic resonance imaging detection with gradient tensor compensation in urban unshielded environment, Appl. Phys. Lett. 102 (2013) 102602.

[21] C. Liu, B. Chang, L. Qiu, Y. Qiu, H. Dong, Y. Zhang, X. Xie, Multichannel ULF-MRI study in magnetic unshielded urban laboratory environment, IEEE Trans. Appl. Supercond. 25 (2015) $1-4$

[22] J.L. Paulsen, L.S. Bouchard, D. Graziani, B. Blümich, A. Pines, Volume-selective magnetic resonance imaging using an adjustable, single-sided, portable sensor, Proc. Natl. Acad. Sci. USA. 105 (2008) 20601-20604.

[23] B. Inglis, K. Buckenmaier, P. SanGiorgio, A.F. Pedersen, M.A. Nichols, J. Clarke, MRI of the human brain at 130 microtesla. Proc. Natl. Acad. Sci. USA. 110 (2013) 19194-19201.

[24] M. Sarracanie, C.D. LaPierre, N. Salameh, D.E.J. Waddington, T. Witzel, M.S. Rosen, Low- 
cost high-performance MRI, Sci. Rep. 5 (2015) 15177.

[25] R.W. Mair, M.I. Hrovat, S. Patz, M.S. Rosen, I.C. Ruset, G.P. Topulos, L.L. Tsai, J.P. Butler, F.W. Hersman, R.L. Walsworth, ${ }^{3} \mathrm{He}$ lung imaging in an open access, very-low-field human magnetic resonance imaging system, Magn. Reson. Med. 53 (2005) 745-749.

[26] J. Lane, J. Datta, B. Karley, J. Norwood, DSP Filters (Indianapolis: Prompt Press), 2000. pp. 205-222.

[27] B. Widrow, J.R. Glover, J. M. McCool, J. Kaunitz, C.S. Williams, R.H. Hearn, J.R. Zeidler, J.R. Eugene, R.C. Goodlin, Adaptive noise cancelling: principles and applications. Proc. IEEE 63 (1975) 1692-1716.

[28] P. Jiruska, R. Cmejla, A.D. Powell ,W. Chang, M. Vreugdenhil, J.G.R. Jefferys, Reference noise method of removing powerline noise from recorded signals, J. Neurosci. Meth. 184 (2009) 110-114.

[29] A. Legchenko, P. Valla, Removal of power-line harmonics from proton magnetic resonance measurements, J. Appl. Geophys. 53 (2003) 103-120.

[30] J. Vrba, Multichannel SQUID biomagnetic systems Application of Superconductivity ed H. Weinstok (Dordrecht: Kluwer-Academic), 2000, pp. 61-138

[31] A. Matlashov, P. Magnelind, S. Newman, H. Sandin, A. Urbaitis, P. Volegov, M. Espy, Multichannel SQUID-based ultra-low field magnetic resonance imaging in unshielded environment, in: 15th International Superconductive Electronics Conference, IEEE, 2015.

[32] J. Clarke, A.I. Braginski, The SQUID Handbook: Fundamentals and Technology of SQUIDs and SQUID Systems (Weinheim: Wiley Online Library), Volume I. 2004

[33] M. Karam, H.F. Khazaal, H. Aglan, C. Cole, Noise removal in speech processing using spectral subtraction, J. Sig. Info. Proc. 5 (2014) 32-41.

[34] L. Debnath, Wavelet Transforms and Their Applications (Basel: Birkhäuser), 2002, pp. 685713.

[35] K. Bartušek, J. Přinosil, Z. Smékal, Wavelet-based de-noising techniques in MRI, Comput. Meth. Prog. Bio. 104 (2001):480-488.

[36] D. Brunet, E. R.Vrscay, Z. Wang, The use of residuals in image denoising, Comput. Notes. Comput. Sc. 5627 (2009): 1-12. 